\title{
Acceptance Sampling Plans from Life Tests Based on Percentiles of Half Normal Distribution
}

\author{
B. Srinivasa Rao, ${ }^{1}$ Ch. Srinivasa Kumar, ${ }^{2}$ and K. Rosaiah $^{3}$ \\ ${ }^{1}$ Department of Mathematics \& Humanities, R.V.R \& J.C College of Engineering, Guntur, Aandhra Pardesh 522 019, India \\ ${ }^{2}$ Department of Mathematics, K.L.University, Guntur, Aandhra Pardesh 522 502, India \\ ${ }^{3}$ Department of Statistics, Acharya Nagarjuna University, Guntur, Aandhra Pardesh 522 010, India
}

Correspondence should be addressed to B. Srinivasa Rao; boyapatisrinu@yahoo.com

Received 15 April 2013; Revised 9 October 2013; Accepted 11 October 2013

Academic Editor: Christian Kirchsteiger

Copyright (C) 2013 B. Srinivasa Rao et al. This is an open access article distributed under the Creative Commons Attribution License, which permits unrestricted use, distribution, and reproduction in any medium, provided the original work is properly cited.

\begin{abstract}
The design of acceptance sampling plans is developed under truncated life testing based on the percentiles of half normal distribution. The minimum sample size necessary to ensure the specified life percentile is obtained under a given consumer's risk. The operating characteristic values of the sampling plans as well as the producer's risk are presented. The results are illustrated by examples.
\end{abstract}

\section{Introduction}

Acceptance sampling is concerned with inspection and decision making regarding lots of products and constitutes one of the oldest techniques in quality control. If the lifetime of the product represents the quality characteristics of interest, the acceptance sampling is as follows: a company receives a shipment of product from a vendor. This product is often a component or raw material used in the company's manufacturing process. A sample is taken from the lot and the relevant quality characteristic of the units in the sample is inspected. On the basis of the information in the sample, a decision is made regarding lot disposition. Traditionally, when the life test indicates that the mean life of products exceeds the specified one, the lot of products is accepted, otherwise it is rejected. Accepted lots are put into production, while rejected lots may be returned to the vendor or may be subjected to some other lot disposition actions. For the purpose of reducing the test time and cost, a truncated life test may be conducted to determine the sample size to ensure a certain mean life of products when the life test is terminated at a time $t_{0}$, and the number of failures does not exceed a given acceptance number $c$.

A common practice in life testing is to terminate the life test by a predetermined time $t_{0}$ and note the number of failures. One of the objectives of these experiments is to set a lower confidence limit on the mean life. It is then to establish a specified mean life with a given probability of at least $p^{*}$ which provides protection to consumers. The test may be terminated before the time is reached or when the number of failures exceeds the acceptance number $c$ in which case the decision is to reject the lot.

Studies regarding truncated life tests can be found in Epstein [1], Sobel and Tischendrof [2], Goode and Kao [3], Gupta and Groll [4], Gupta [5], Fertig and Mann [6], Kantam and Rosaiah [7], Baklizi [8], Wu and Tsai [9], Rosaiah and Kantam [10], Rosaiah et al. [11], Tsai and $\mathrm{Wu}$ [12], Balakrishnan et al. [13], Srinivasa Rao et al. [14], Srinivasa Rao et al. [15], Aslam et al. [16], and Srinivasa Rao et al. [17]. All these authors designed acceptance sampling plans based on the mean life time under a truncated life test. In contrast, Lio et al. [18] considered acceptance sampling plans for percentiles using truncated life tests and assuming Birnbaum-Saunders distribution. Srinivasa Rao and Kantam [19] developed similar plans for the percentiles of log-logistic distribution.

Normal distribution is the most preferred distribution in statistical studies. But for life test models it is not suitable distribution because of its domain $[-\infty,+\infty]$. Half normal distribution is an increasing failure rate (IFR) model which is most useful in reliability studies. Because of this IFR nature with domain $[0,+\infty]$ we are motivated to study this distribution. 
In this paper, acceptance sampling plans are developed for percentiles of half normal distribution life test and are given in Section 2. Operating characteristic (OC) and producer's risk are given in Section 3. Examples based on real fatigue life data sets are provided for an illustration in Section 4. The paper is closed with summary and conclusions in Section 5.

\section{Acceptance Sampling Plans}

The probability density function (pdf) of a half normal distribution is given by

$$
p(x)=\frac{2 \theta}{\pi} e^{-x^{2} \theta^{2} / \pi}, \quad x \geq 0 .
$$

Its cumulative distribution function (cdf) is

$$
P(x)=\operatorname{erf}\left(\frac{\theta x}{\sqrt{\pi}}\right), \quad x \geq 0,
$$

where erf is an error function and $\theta$ is a parameter. Assume that the life time of a product follows half normal distribution with $\sigma$ as scale parameter. Its cumulative distribution function $F(\cdot)$ is given by

$$
F(t)=\operatorname{erf}\left(\frac{\theta(t / \sigma)}{\sqrt{\pi}}\right), \quad t \geq 0, \sigma>0
$$

Given $0<q<1$, the 100th percentile is given by

$$
t_{q}=\sigma \frac{\sqrt{\pi}}{\theta} \operatorname{erf}^{-1}(q)
$$

Substituting $\sigma$ in (3) in the scaled form we get

$$
\begin{aligned}
F(t) & =\operatorname{erf}\left(\frac{\theta\left(t / t_{q}\right)(\sqrt{\pi} / \theta) \operatorname{erf}^{-1}(q)}{\sqrt{\pi}}\right) \\
& \Longrightarrow \operatorname{erf}\left(\delta_{0} \operatorname{erf}^{-1}(q)\right)
\end{aligned}
$$

where $\delta_{0}=t / t_{q}$. Here $\operatorname{erf}^{-1}$ is simulated by

$$
\begin{aligned}
\operatorname{erf}^{-1}(z)=\frac{1}{2} \sqrt{\pi}[z & +\frac{\pi}{12} z^{3}+\frac{7 \pi^{2}}{480} z^{5} \\
& \left.+\frac{127 \pi^{3}}{40320} z^{7}+\frac{4369 \pi^{4}}{5806080} z^{9} \cdots\right]
\end{aligned}
$$

A common practice in life testing is to terminate the life test by a predetermined time $t$, to require the probability of rejecting a bad lot to be at least $p^{*}$, and to have the maximum permissible number of bad items to accept the lot equal to $c$. The acceptance sampling plan for percentiles under a truncated life test is to set up the minimum sample size $n$ for a given acceptance number $c$ such that the consumer's risk, the probability of accepting a bad lot, does not exceed $1-p^{*}$. A bad lot means that the true 100th percentile, $t_{q}$, is below a specified percentile, $t_{q}^{0}$. Thus the probability $p^{*}$ is a confidence level in the sense that the chance of rejecting a bad lot with $t_{q}<t_{q}^{0}$ is at least equal to $p^{*}$. Therefore, for given $p^{*}$, the proposed acceptance sampling plan can be characterized by the triplet $\left(n, c, \delta_{0}\right)=\left(n, c, t / t_{q}^{0}\right)$, where $\delta=t / t_{q}^{0}$.

We consider large sized lots so that the binomial distribution can be applied. The problem is to determine for given values of $p^{*}, t_{q}^{0}$, and $c$ the smallest positive integer $n$ required to assert that $t_{q}>t_{q}^{0}$ must satisfy the relation

$$
\sum_{i=0}^{c} p_{0}^{i}\left(1-p_{0}\right)^{n-i} \leq 1-p^{*}
$$

where $p_{0}=F_{t}\left(\delta_{0}\right)$ is the probability of a failure during the time $t=\delta t_{q}^{0}$ for the specified 100qth percentile of lifetime $t_{q}^{0}$. The value $p_{0}$ depends only on $\delta_{0}=t / t_{q}^{0}$. Since $\partial F_{t}(\delta) / \partial \delta>0$, it is an increasing function of $\delta$. Accordingly, we have

$$
F_{t}(\delta) \leq F_{t}\left(\delta_{0}\right) \Longleftrightarrow \delta \leq \delta_{0}
$$

or equivalently

$$
F\left(t ; t_{q}\right)<F\left(t ; t_{q}^{0}\right) \Longleftrightarrow t_{q} \geq t_{q}^{0}
$$

The smallest sample size $n$ satisfying (9) can be obtained for any given $q, t / t_{q}^{0}$, or $p^{*}$. In contrast, only the input values $t / \sigma, p^{*}$ are needed to calculate the smallest sample size $n$. In particular, if we take $q=0.50$, the value of $n$ would become "the smallest sample size required to test that the population median life exceeds a given specified value." Half normal distribution is a skewed distribution; for our present skewed population the median is a more appropriate average for decision making about the quality of the life than population mean. Thus we may conclude that population median based sampling plans of half normal distribution model are more economical than those based on population mean with respect to sample size. To save space, only the results of small sample sizes for $q=0.50 ; p^{*}=0.75,0.90,0.95,0.99 ; c=$ $0(1) 10 ; \delta=0.1,0.2,0.4,0.6,0.8,1.0,1.5,2.0,2.5,3.0$ are displayed in Table 1 .

\section{Operating Characteristic of the Sampling Plan and Producer's Risk}

The operating characteristic (OC) function $L$ of the sampling plan $\left(n, c, t / t_{q}^{0}\right)$ is the probability of accepting a lot as a function of $p=F_{t}(\delta)$ with $\delta_{0}=t / t_{q}$. It is given as

$$
L(p)=\sum_{i=0}^{c}\left(\begin{array}{c}
n \\
i
\end{array}\right) p^{i}(1-p)^{n-i}
$$

Therefore, we have

$$
p=F_{t}(\delta)=F\left(\frac{t}{t_{q}^{0}} \frac{1}{d_{q}}\right),
$$

where $d_{q}=t_{q} / t_{q}^{0}$. Using (10) the OC values can be obtained for any sampling plan $\left(n, c, t / t_{0.5}^{0}\right)$ (Table 5$)$. To save space we 
TABLE 1: Minimum sample sizes necessary to assert the median life of a product.

\begin{tabular}{|c|c|c|c|c|c|c|c|c|c|c|c|}
\hline \multirow{2}{*}{$p^{*}$} & \multirow{2}{*}{$c$} & \multicolumn{10}{|c|}{$t / t_{0.5}^{0}$} \\
\hline & & 0.1 & 0.2 & 0.4 & 0.6 & 0.8 & 1.0 & 1.5 & 2.0 & 2.5 & 3.0 \\
\hline 0.75 & 0 & 26 & 13 & 6 & 4 & 3 & 3 & 2 & 2 & 2 & 1 \\
\hline 0.75 & 1 & 50 & 25 & 12 & 8 & 6 & 5 & 3 & 3 & 2 & 2 \\
\hline 0.75 & 2 & 72 & 36 & 18 & 12 & 9 & 7 & 5 & 4 & 4 & 3 \\
\hline 0.75 & 3 & 94 & 47 & 23 & 16 & 12 & 10 & 7 & 5 & 5 & 4 \\
\hline 0.75 & 4 & 116 & 58 & 29 & 19 & 15 & 12 & 8 & 7 & 6 & 5 \\
\hline 0.75 & 5 & 137 & 68 & 34 & 23 & 17 & 14 & 10 & 8 & 7 & 6 \\
\hline 0.75 & 6 & 158 & 79 & 39 & 26 & 20 & 16 & 11 & 9 & 8 & 8 \\
\hline 0.75 & 7 & 179 & 89 & 45 & 30 & 23 & 18 & 13 & 11 & 9 & 9 \\
\hline 0.75 & 8 & 200 & 100 & 50 & 33 & 25 & 21 & 14 & 12 & 10 & 10 \\
\hline 0.75 & 9 & 220 & 110 & 55 & 37 & 28 & 23 & 16 & 13 & 12 & 11 \\
\hline 0.75 & 10 & 241 & 120 & 60 & 40 & 31 & 25 & 18 & 14 & 13 & 12 \\
\hline 0.90 & 0 & 42 & 21 & 10 & 7 & 5 & 4 & 2 & 2 & 2 & 2 \\
\hline 0.90 & 1 & 71 & 35 & 17 & 11 & 8 & 7 & 4 & 3 & 3 & 2 \\
\hline 0.90 & 2 & 98 & 48 & 24 & 16 & 12 & 9 & 6 & 5 & 4 & 4 \\
\hline 0.90 & 3 & 123 & 61 & 30 & 20 & 15 & 12 & 8 & 6 & 5 & 5 \\
\hline 0.90 & 4 & 147 & 73 & 36 & 24 & 18 & 14 & 10 & 8 & 6 & 6 \\
\hline 0.90 & 5 & 171 & 85 & 42 & 28 & 21 & 17 & 11 & 9 & 8 & 7 \\
\hline 0.90 & 6 & 194 & 96 & 48 & 32 & 24 & 19 & 13 & 10 & 9 & 8 \\
\hline 0.90 & 7 & 217 & 108 & 53 & 35 & 27 & 21 & 15 & 12 & 10 & 9 \\
\hline 0.90 & 8 & 240 & 119 & 59 & 39 & 29 & 24 & 16 & 13 & 11 & 10 \\
\hline 0.90 & 9 & 262 & 130 & 65 & 43 & 32 & 26 & 18 & 14 & 12 & 11 \\
\hline 0.90 & 10 & 284 & 141 & 70 & 47 & 35 & 28 & 20 & 16 & 14 & 12 \\
\hline 0.95 & 0 & 55 & 27 & 13 & 8 & 6 & 5 & 3 & 2 & 2 & 2 \\
\hline 0.95 & 1 & 87 & 43 & 21 & 14 & 10 & 8 & 5 & 4 & 3 & 3 \\
\hline 0.95 & 2 & 115 & 57 & 28 & 18 & 13 & 11 & 7 & 5 & 4 & 4 \\
\hline 0.95 & 3 & 142 & 70 & 34 & 23 & 17 & 13 & 9 & 7 & 6 & 5 \\
\hline 0.95 & 4 & 168 & 83 & 41 & 27 & 20 & 16 & 11 & 8 & 7 & 6 \\
\hline 0.95 & 5 & 193 & 96 & 47 & 31 & 23 & 18 & 12 & 10 & 8 & 7 \\
\hline 0.95 & 6 & 218 & 108 & 53 & 35 & 26 & 21 & 14 & 11 & 9 & 8 \\
\hline 0.95 & 7 & 242 & 120 & 59 & 39 & 29 & 23 & 16 & 12 & 11 & 10 \\
\hline 0.95 & 8 & 266 & 132 & 65 & 43 & 32 & 26 & 18 & 14 & 12 & 11 \\
\hline 0.95 & 9 & 289 & 143 & 71 & 47 & 35 & 28 & 19 & 15 & 13 & 12 \\
\hline 0.95 & 10 & 312 & 155 & 77 & 51 & 38 & 30 & 21 & 16 & 14 & 13 \\
\hline 0.99 & 0 & 84 & 41 & 20 & 13 & 9 & 7 & 4 & 3 & 2 & 2 \\
\hline 0.99 & 1 & 121 & 59 & 29 & 19 & 14 & 11 & 7 & 5 & 4 & 3 \\
\hline 0.99 & 2 & 154 & 76 & 37 & 24 & 17 & 14 & 9 & 7 & 5 & 5 \\
\hline 0.99 & 3 & 184 & 91 & 44 & 29 & 21 & 17 & 11 & 8 & 7 & 6 \\
\hline 0.99 & 4 & 212 & 105 & 51 & 33 & 25 & 19 & 13 & 10 & 8 & 7 \\
\hline 0.99 & 5 & 240 & 119 & 58 & 38 & 28 & 22 & 15 & 11 & 9 & 8 \\
\hline 0.99 & 6 & 267 & 132 & 65 & 42 & 31 & 25 & 16 & 13 & 10 & 9 \\
\hline 0.99 & 7 & 293 & 145 & 71 & 47 & 35 & 27 & 18 & 14 & 12 & 10 \\
\hline 0.99 & 8 & 319 & 158 & 77 & 51 & 38 & 30 & 20 & 15 & 13 & 12 \\
\hline 0.99 & 9 & 345 & 171 & 84 & 55 & 41 & 33 & 22 & 17 & 14 & 13 \\
\hline 0.99 & 10 & 370 & 183 & 90 & 59 & 44 & 35 & 24 & 18 & 15 & 14 \\
\hline
\end{tabular}

present the OC values for sampling plans with $q=0.50 ; p^{*}=$ $0.75,0.90,0.95,0.99 ; \delta=0.1,0.2,0.4,0.6,0.8,1.0,1.5,2.0,2.5,3.0$; $t_{0.5} / t_{0.5}^{0}=1.0,1.5,2.0,2.5,3.0,3.5,4.0,4.5,5.0$; for $c=1$ and $c=5$ which are given in Table 2 and Table 3 , respectively.
The producer's risk is defined as the probability of rejecting the lot when $t_{q}>t_{q}^{0}$. For a given value of the producer's risk, say $\gamma$, we are interested in knowing the value of $d_{q}$ to ensure that the producer's risk is less than or equal to $\gamma$ if a sampling plan $\left(n, c, t / t_{q}\right)$ is developed at a specified confidence level $p^{*}$. Thus, one needs to find the smallest value $d_{q}$ according to (10) as

$$
\sum_{i=0}^{c}\left(\begin{array}{c}
n \\
i
\end{array}\right) p^{i}(1-p)^{n-i}
$$

where

$$
p=F\left(\frac{t}{t_{q}^{0}} \frac{1}{d_{q}}\right)
$$

To save space, based on the sampling plans $\left(n, c, t / t_{q}^{0}\right)$ given in Table 1 the minimum ratios of $d_{0.5}$ for the acceptability of a lot at the producer's risk of $\gamma=0.05$ are presented in Table 4 .

\section{Illustrative Examples}

In this section, we consider two examples with real data sets to illustrate the proposed acceptance sampling plans.

4.1. Example 1. The first data refers to software reliability presented by Wood [20]. The data set was reported in hours as $519,968,1430,1893,2490,3058,3625,4422$, and 5218 . The confidence level is assumed for this acceptance sampling plan only if the lifetimes are from half normal distribution with shape parameter $\sigma$. So in order to apply this example for our tables we have to confirm the goodness of fit of half normal distribution to the data in the example. This is done through the well-known QQ-plot method, and the value we get is $R=$ 0.9287 . Hence we conclude that the half normal distribution provides a reasonable goodness of fit for the data.

Suppose that the experimenter would like to establish the unknown 50th percentile life time for the software mentioned above to be at least $300 \mathrm{~h}$ and the life test would be ended at $600 \mathrm{~h}$ which should have led to the ratio $t / t_{0.5}^{0}=2.0$. Thus, with $c=1$ and $p^{*}=0.75$, the experimenter may take from Table 1 the sample size $n$ which must be at least 3 ; thus the sampling plan to the data is given by

$$
\left(n, c, \frac{t}{t_{0.5}^{0}}\right)=(3,1,2.0) \text {. }
$$

Since there is one item with a failure time less than or equal to $600 \mathrm{~h}$ in the given sample of 9 observations, the lot is accepted as the result indicates that the 50th percentile life time $t_{0.5}$ is at least $300 \mathrm{~h}$ with a confidence level of $p^{*}=0.75$.

The OC values for acceptance sampling plan in (14) and confidence $p^{*}=0.75$, for a half normal distribution, may be taken from Table 2.

The producer's risk is almost equal to $0.3683(=1-$ 0.6317 ), when the true percentile is greater than or equal to 2.0 times the specified 50th percentile. 
TABLE 2: OC values of sampling plans.

\begin{tabular}{|c|c|c|c|c|c|c|c|c|c|c|c|}
\hline \multirow{2}{*}{$\begin{array}{l}c=1 \\
p^{*}\end{array}$} & \multicolumn{11}{|c|}{$t / t_{0.5}^{0}$} \\
\hline & $n$ & $\delta_{0.01}$ & 1.0 & 1.5 & 2.0 & 2.5 & 3.0 & 3.5 & 4.0 & 4.5 & 5.0 \\
\hline \multirow{10}{*}{0.75} & 50 & 0.1 & 0.2420 & 0.4605 & 0.6093 & 0.7075 & 0.7741 & 0.8207 & 0.8545 & 0.8796 & 0.8989 \\
\hline & 25 & 0.2 & 0.2345 & 0.4576 & 0.6090 & 0.7084 & 0.7754 & 0.8221 & 0.8559 & 0.8810 & 0.9001 \\
\hline & 12 & 0.4 & 0.2408 & 0.4764 & 0.6301 & 0.7281 & 0.7928 & 0.8373 & 0.8690 & 0.8924 & 0.9101 \\
\hline & 8 & 0.6 & 0.2283 & 0.4759 & 0.6347 & 0.7342 & 0.7989 & 0.8430 & 0.8742 & 0.8970 & 0.9142 \\
\hline & 6 & 0.8 & 0.2173 & 0.4788 & 0.6425 & 0.7427 & 0.8070 & 0.8502 & 0.8805 & 0.9026 & 0.9191 \\
\hline & 5 & 1.0 & 0.1875 & 0.4609 & 0.6328 & 0.7373 & 0.8038 & 0.8482 & 0.8793 & 0.9018 & 0.9185 \\
\hline & 3 & 1.5 & 0.2309 & 0.5616 & 0.7262 & 0.8143 & 0.8662 & 0.8992 & 0.9214 & 0.9370 & 0.9484 \\
\hline & 3 & 2.0 & 0.0832 & 0.4276 & 0.6317 & 0.7464 & 0.8157 & 0.8602 & 0.8905 & 0.9120 & 0.9272 \\
\hline & 2 & 2.5 & 0.1752 & 0.6334 & 0.7938 & 0.8680 & 0.9084 & 0.9327 & 0.9484 & 0.9593 & 0.9670 \\
\hline & 2 & 3.0 & 0.0843 & 0.5920 & 0.7711 & 0.8535 & 0.8983 & 0.9253 & 0.9428 & 0.9548 & 0.9634 \\
\hline \multirow{10}{*}{0.90} & 71 & 0.1 & 0.0993 & 0.2723 & 0.4274 & 0.5466 & 0.6354 & 0.7019 & 0.7524 & 0.7915 & 0.8221 \\
\hline & 35 & 0.2 & 0.0980 & 0.2752 & 0.4332 & 0.5534 & 0.6424 & 0.7085 & 0.7586 & 0.7971 & 0.8272 \\
\hline & 17 & 0.4 & 0.0961 & 0.2833 & 0.4473 & 0.5695 & 0.6583 & 0.7235 & 0.7723 & 0.8095 & 0.8384 \\
\hline & 11 & 0.6 & 0.0953 & 0.2950 & 0.4654 & 0.5891 & 0.6772 & 0.7409 & 0.7879 & 0.8235 & 0.8509 \\
\hline & 8 & 0.8 & 0.0958 & 0.3109 & 0.4880 & 0.6124 & 0.6989 & 0.7604 & 0.8053 & 0.8388 & 0.8645 \\
\hline & 7 & 1.0 & 0.0625 & 0.2634 & 0.4449 & 0.5767 & 0.6698 & 0.7365 & 0.7854 & 0.8221 & 0.8503 \\
\hline & 4 & 1.5 & 0.0928 & 0.3766 & 0.5734 & 0.6949 & 0.7725 & 0.8243 & 0.8605 & 0.8866 & 0.9061 \\
\hline & 3 & 2.0 & 0.0832 & 0.4276 & 0.6317 & 0.7464 & 0.8157 & 0.8602 & 0.8905 & 0.9120 & 0.9277 \\
\hline & 3 & 2.5 & 0.0237 & 0.3441 & 0.5687 & 0.7000 & 0.7805 & 0.8329 & 0.8688 & 0.8942 & 0.9130 \\
\hline & 2 & 3.0 & 0.0843 & 0.5930 & 0.7711 & 0.8535 & 0.8983 & 0.9253 & 0.9428 & 0.9548 & 0.9364 \\
\hline \multirow{10}{*}{0.95} & 87 & 0.1 & 0.0484 & 0.1766 & 0.3176 & 0.4390 & 0.5362 & 0.6127 & 0.6730 & 0.7209 & 0.7594 \\
\hline & 43 & 0.2 & 0.0468 & 0.1773 & 0.3210 & 0.4440 & 0.5420 & 0.6186 & 0.6788 & 0.7264 & 0.7645 \\
\hline & 21 & 0.4 & 0.0441 & 0.1804 & 0.3302 & 0.4566 & 0.5558 & 0.6325 & 0.6920 & 0.7388 & 0.7760 \\
\hline & 14 & 0.6 & 0.0377 & 0.1753 & 0.3298 & 0.4595 & 0.5605 & 0.6380 & 0.6978 & 0.7445 & 0.7815 \\
\hline & 10 & 0.8 & 0.0404 & 0.1948 & 0.3602 & 0.4932 & 0.5935 & 0.6688 & 0.7259 & 0.7699 & 0.8043 \\
\hline & 8 & 1.0 & 0.0352 & 0.1951 & 0.3671 & 0.5033 & 0.6047 & 0.6798 & 0.7363 & 0.7795 & 0.8131 \\
\hline & 5 & 1.5 & 0.0354 & 0.2431 & 0.4397 & 0.5795 & 0.6761 & 0.7441 & 0.7933 & 0.8297 & 0.8575 \\
\hline & 4 & 2.0 & 0.0194 & 0.2436 & 0.4558 & 0.6002 & 0.6969 & 0.7633 & 0.8105 & 0.8450 & 0.8710 \\
\hline & 3 & 2.5 & 0.0237 & 0.3441 & 0.5687 & 0.7000 & 0.7805 & 0.8329 & 0.8688 & 0.8942 & 0.9130 \\
\hline & 3 & 3.0 & 0.0054 & 0.2983 & 0.5323 & 0.6726 & 0.7597 & 0.8166 & 0.8557 & 0.8836 & 0.9041 \\
\hline \multirow{10}{*}{0.99} & 121 & 0.1 & 0.0098 & 0.0662 & 0.1603 & 0.2633 & 0.3593 & 0.4433 & 0.5148 & 0.5749 & 0.6256 \\
\hline & 59 & 0.2 & 0.0100 & 0.0695 & 0.1679 & 0.2740 & 0.3718 & 0.4565 & 0.5280 & 0.5878 & 0.6378 \\
\hline & 29 & 0.4 & 0.0086 & 0.0688 & 0.1709 & 0.2807 & 0.3811 & 0.4672 & 0.5392 & 0.5991 & 0.6489 \\
\hline & 19 & 0.6 & 0.0075 & 0.0694 & 0.1766 & 0.2908 & 0.3938 & 0.4812 & 0.5536 & 0.6133 & 0.6626 \\
\hline & 14 & 0.8 & 0.0066 & 0.0714 & 0.1851 & 0.3044 & 0.4103 & 0.4987 & 0.5712 & 0.6303 & 0.6788 \\
\hline & 11 & 1.0 & 0.0059 & 0.0751 & 0.1971 & 0.3221 & 0.4307 & 0.5199 & 0.5919 & 0.6501 & 0.6974 \\
\hline & 7 & 1.5 & 0.0047 & 0.0942 & 0.2439 & 0.3841 & 0.4975 & 0.5859 & 0.6446 & 0.7083 & 0.7509 \\
\hline & 5 & 2.0 & 0.0042 & 0.1328 & 0.3177 & 0.4694 & 0.5819 & 0.6644 & 0.7257 & 0.7721 & 0.8079 \\
\hline & 4 & 2.5 & 0.0029 & 0.1730 & 0.3843 & 0.5395 & 0.6469 & 0.7222 & 0.7764 & 0.8164 & 0.8467 \\
\hline & 3 & 3.0 & 0.0054 & 0.2983 & 0.5323 & 0.6726 & 0.7597 & 0.8166 & 0.8557 & 0.8836 & 0.9041 \\
\hline
\end{tabular}

From Table 4, the experimenter could get the values of $d_{0.5}$ for different choices of $c$ and $t / t_{0.5}^{0}$ in order to assert that the producer's risk is less than 0.05 . In this example, the value of $d_{0.5}$ should be 7.9120 for $c=1, t_{0.5} / t_{0.5}^{0}=2.0$, and $p^{*}=0.75$. This means that the product can have a life of 7.9120 times the required 50th percentile life time in order that under the above acceptance sampling plan the product is accepted with probability at least 0.75 .
4.2. Example 2. The second data refers to the data obtained from Aarset [21]. It represents the lifetimes of 50 devices in hours. The data set was reported in hours as $0.1,0.2,1,1,1,1$, $1,2,3,6,7,11,12,18,18,18,18,18,21,32,36,40,45,46,47,50$, $55,60,63,63,67,67,67,67,72,75,79,82,82,83,84,84,84,85$, $85,85,85,85,86$, and 86 . On the similar lines of Example 1 , for this data, the goodness of fit for 50 observations showed that $R=0.9175$. 
TABLE 3: OC values of sampling plans.

\begin{tabular}{|c|c|c|c|c|c|c|c|c|c|c|c|}
\hline \multirow{2}{*}{$\begin{array}{l}c=5 \\
p^{*}\end{array}$} & \multicolumn{11}{|c|}{$t / t_{0.5}^{0}$} \\
\hline & $n$ & $\delta_{0.01}$ & 1.0 & 1.5 & 2.0 & 2.5 & 3.0 & 3.5 & 4.0 & 4.5 & 5.0 \\
\hline \multirow{10}{*}{0.75} & 137 & 0.1 & 0.2484 & 0.6314 & 0.8348 & 0.9234 & 0.9623 & 0.9802 & 0.9891 & 0.9936 & 0.9961 \\
\hline & 68 & 0.2 & 0.2493 & 0.6407 & 0.8427 & 0.9284 & 0.9652 & 0.9820 & 0.9901 & 0.9943 & 0.9966 \\
\hline & 34 & 0.4 & 0.2408 & 0.6506 & 0.8533 & 0.9354 & 0.9694 & 0.9845 & 0.9916 & 0.9952 & 0.9972 \\
\hline & 23 & 0.6 & 0.2228 & 0.6527 & 0.8596 & 0.9400 & 0.9723 & 0.9862 & 0.9926 & 0.9959 & 0.9976 \\
\hline & 17 & 0.8 & 0.2359 & 0.6888 & 0.8833 & 0.9527 & 0.9790 & 0.9898 & 0.9947 & 0.9971 & 0.9983 \\
\hline & 14 & 1.0 & 0.2120 & 0.6898 & 0.8883 & 0.9561 & 0.9809 & 0.9909 & 0.9953 & 0.9974 & 0.9985 \\
\hline & 10 & 1.5 & 0.1711 & 0.7193 & 0.9118 & 0.9685 & 0.9871 & 0.9942 & 0.9971 & 0.9985 & 0.9991 \\
\hline & 8 & 2.0 & 0.1556 & 0.7826 & 0.9428 & 0.9816 & 0.9930 & 0.9970 & 0.9986 & 0.9993 & 0.9996 \\
\hline & 7 & 2.5 & 0.1297 & 0.8341 & 0.9625 & 0.9889 & 0.9960 & 0.9983 & 0.9992 & 0.9996 & 0.9998 \\
\hline & 6 & 3.0 & 0.2323 & 0.9326 & 0.9880 & 0.9969 & 0.9989 & 0.9996 & 0.9998 & 0.9999 & 1.0000 \\
\hline \multirow{10}{*}{0.90} & 171 & 0.1 & 0.0008 & 0.0143 & 0.0541 & 0.1154 & 0.1868 & 0.2595 & 0.3288 & 0.3925 & 0.4497 \\
\hline & 85 & 0.2 & 0.0007 & 0.0137 & 0.0536 & 0.1156 & 0.1879 & 0.2615 & 0.3314 & 0.3955 & 0.4531 \\
\hline & 42 & 0.4 & 0.0005 & 0.0129 & 0.0534 & 0.1173 & 0.1918 & 0.2673 & 0.3387 & 0.4038 & 0.4619 \\
\hline & 28 & 0.6 & 0.0004 & 0.0117 & 0.0519 & 0.1169 & 0.1930 & 0.2701 & 0.3428 & 0.4087 & 0.4675 \\
\hline & 21 & 0.8 & 0.0002 & 0.0108 & 0.0516 & 0.1185 & 0.1970 & 0.2760 & 0.3501 & 0.4170 & 0.4763 \\
\hline & 17 & 1.0 & 0.0001 & 0.0096 & 0.0501 & 0.1182 & 0.1983 & 0.2789 & 0.3542 & 0.4219 & 0.4818 \\
\hline & 11 & 1.5 & 0.0001 & 0.0120 & 0.0654 & 0.1499 & 0.2431 & 0.3321 & 0.4117 & 0.4810 & 0.5404 \\
\hline & 9 & 2.0 & 0.0000 & 0.0093 & 0.0619 & 0.1492 & 0.2459 & 0.3377 & 0.4193 & 0.4898 & 0.5499 \\
\hline & 8 & 2.5 & 0.0000 & 0.0078 & 0.0604 & 0.1503 & 0.2499 & 0.3439 & 0.4268 & 0.4980 & 0.5584 \\
\hline & 7 & 3.0 & 0.0000 & 0.0109 & 0.0779 & 0.1823 & 0.2907 & 0.3885 & 0.4722 & 0.5422 & 0.6006 \\
\hline \multirow{10}{*}{0.95} & 193 & 0.1 & 0.0003 & 0.0071 & 0.0328 & 0.0787 & 0.1376 & 0.2019 & 0.2661 & 0.3273 & 0.3841 \\
\hline & 96 & 0.2 & 0.0002 & 0.0068 & 0.0324 & 0.0787 & 0.1382 & 0.2031 & 0.2680 & 0.3298 & 0.3869 \\
\hline & 47 & 0.4 & 0.0002 & 0.0067 & 0.0335 & 0.0824 & 0.1449 & 0.2124 & 0.2793 & 0.3425 & 0.4006 \\
\hline & 31 & 0.6 & 0.0001 & 0.0063 & 0.0339 & 0.0849 & 0.1499 & 0.2197 & 0.2884 & 0.3529 & 0.4117 \\
\hline & 23 & 0.8 & 0.0001 & 0.0062 & 0.0352 & 0.0892 & 0.1575 & 0.2301 & 0.3008 & 0.3666 & 0.4262 \\
\hline & 18 & 1.0 & 0.0001 & 0.0068 & 0.0395 & 0.0991 & 0.1728 & 0.2495 & 0.3228 & 0.3901 & 0.4503 \\
\hline & 12 & 1.5 & 0.0000 & 0.0070 & 0.0462 & 0.1166 & 0.2004 & 0.2845 & 0.3624 & 0.4320 & 0.4930 \\
\hline & 10 & 2.0 & 0.0000 & 0.0046 & 0.0399 & 0.1092 & 0.1938 & 0.2794 & 0.3590 & 0.4299 & 0.4920 \\
\hline & 8 & 2.5 & 0.0000 & 0.0078 & 0.0604 & 0.1503 & 0.2499 & 0.3439 & 0.4268 & 0.4980 & 0.5584 \\
\hline & 7 & 3.0 & 0.0000 & 0.0109 & 0.0779 & 0.1823 & 0.2907 & 0.3885 & 0.4722 & 0.5422 & 0.6006 \\
\hline \multirow{10}{*}{0.99} & 240 & 0.1 & 0.0000 & 0.0015 & 0.0110 & 0.0339 & 0.0700 & 0.1153 & 0.1657 & 0.2177 & 0.2691 \\
\hline & 119 & 0.2 & 0.0000 & 0.0015 & 0.0109 & 0.0343 & 0.0710 & 0.1172 & 0.1683 & 0.2211 & 0.2731 \\
\hline & 58 & 0.4 & 0.0000 & 0.0015 & 0.0117 & 0.0369 & 0.0764 & 0.1254 & 0.1792 & 0.2341 & 0.2877 \\
\hline & 38 & 0.6 & 0.0000 & 0.0015 & 0.0122 & 0.0393 & 0.0813 & 0.1331 & 0.0189 & 0.0246 & 0.3012 \\
\hline & 28 & 0.8 & 0.0000 & 0.0015 & 0.0132 & 0.0428 & 0.0883 & 0.1435 & 0.2026 & 0.2617 & 0.3183 \\
\hline & 22 & 1.0 & 0.0000 & 0.0016 & 0.0149 & 0.0480 & 0.0978 & 0.1571 & 0.2195 & 0.2810 & 0.3392 \\
\hline & 15 & 1.5 & 0.0000 & 0.0014 & 0.0159 & 0.0535 & 0.1096 & 0.1750 & 0.2424 & 0.3075 & 0.3681 \\
\hline & 11 & 2.0 & 0.0000 & 0.0023 & 0.0256 & 0.0793 & 0.1518 & 0.2299 & 0.3057 & 0.3756 & 0.4383 \\
\hline & 9 & 2.5 & 0.0000 & 0.0034 & 0.0365 & 0.1055 & 0.1912 & 0.2781 & 0.3588 & 0.4307 & 0.4937 \\
\hline & 8 & 3.0 & 0.0000 & 0.0045 & 0.0457 & 0.1256 & 0.2197 & 0.3115 & 0.3945 & 0.4668 & 0.5289 \\
\hline
\end{tabular}

Suppose that we would like to establish the unknown 50th percentile life time for the devices to be at least 10 hours and the life test would be ended at $15 \mathrm{hrs}$ which should have led to the ratio of $t_{0.5} / t_{0.5}^{0}=1.5$. Thus with $c=5, p^{*}=0.99$, the experimenter may take, from Table 1 , the sample size $n$ which must be at least 15 . Thus the sampling plan to the given data is given by $\left(n, c, t / t_{0.5}^{0}\right)=(15,5,1.5)$. Since there are 13 items with a failure time less than or equal to 15 hours in the given sample of 50 observations, the lot is accepted as the result indicates that the 50 th percentile lifetime $t_{0.5}$ is at least 10 hours with a confidence level of $p^{*}=0.99$.

\section{Summary and Conclusions}

This paper provides the minimum sample size required to decide upon accepting/rejecting a lot based on its specified 50 th percentile (median) when the data follows half normal distribution. Assuming that the size of the given sample 
TABLE 4: Minimum ratio for the acceptability of a lot with producer's risk 0.05 .

\begin{tabular}{|c|c|c|c|c|c|c|c|c|c|c|c|}
\hline$p^{*}$ & $c$ & 0.1 & 0.2 & 0.4 & 0.6 & 0.8 & 1.0 & 1.5 & 2.0 & 2.5 & 3.0 \\
\hline 0.75 & 0 & 27.2995 & 27.3264 & 25.2820 & 25.3354 & 25.3887 & 31.7358 & 31.8683 & 42.4911 & 53.1138 & 32.2612 \\
\hline 0.75 & 1 & 7.5211 & 7.4707 & 7.0638 & 6.9551 & 6.8415 & 7.0279 & 5.9340 & 7.9120 & 5.9356 & 7.1227 \\
\hline 0.75 & 2 & 4.6982 & 4.6579 & 4.5740 & 4.4857 & 4.3925 & 4.1606 & 4.2240 & 4.2572 & 5.3215 & 4.2191 \\
\hline 0.75 & 3 & 3.6690 & 3.6354 & 3.4860 & 3.5693 & 3.4898 & 3.5650 & 3.5335 & 3.0410 & 3.8013 & 3.1989 \\
\hline 0.75 & 4 & 3.1398 & 3.1106 & 3.0490 & 2.9280 & 3.0224 & 2.9465 & 2.7276 & 3.0537 & 3.0610 & 2.6281 \\
\hline 0.75 & 5 & 2.7959 & 2.7492 & 2.6938 & 2.6752 & 2.5684 & 2.5816 & 2.5929 & 2.5693 & 2.6240 & 2.3656 \\
\hline 0.75 & 6 & 2.5649 & 2.5411 & 2.4575 & 2.4019 & 2.4074 & 2.3411 & 2.2305 & 2.2542 & 2.3369 & 2.8043 \\
\hline 0.75 & 7 & 2.3985 & 2.3630 & 2.3429 & 2.2910 & 2.2887 & 2.1705 & 2.1968 & 2.3400 & 2.1320 & 2.5584 \\
\hline 0.75 & 8 & 2.2725 & 2.2520 & 2.2076 & 2.1353 & 2.1038 & 2.1610 & 1.9808 & 2.1336 & 1.9782 & 2.3739 \\
\hline 0.75 & 9 & 2.1635 & 2.1442 & 2.1022 & 2.0753 & 2.0432 & 2.0464 & 1.9775 & 1.9748 & 2.1740 & 2.2298 \\
\hline 0.75 & 10 & 2.0847 & 2.0577 & 2.0176 & 1.9725 & 1.9931 & 1.9550 & 1.9712 & 1.8485 & 2.0464 & 2.1137 \\
\hline 0.90 & 0 & 44.0826 & 44.1095 & 42.0653 & 44.2167 & 42.1723 & 42.2257 & 31.8683 & 42.4911 & 53.1138 & 63.7366 \\
\hline 0.90 & 1 & 10.7007 & 10.4994 & 10.0945 & 9.6860 & 9.2735 & 10.0728 & 8.2474 & 7.9120 & 9.8900 & 7.1227 \\
\hline 0.90 & 2 & 6.4092 & 6.2376 & 6.1553 & 6.0698 & 5.9809 & 5.4907 & 5.2368 & 5.6320 & 5.3215 & 6.3857 \\
\hline 0.90 & 3 & 4.8111 & 4.7384 & 4.5906 & 4.5183 & 4.4422 & 4.3623 & 4.1426 & 3.8877 & 3.8013 & 4.5615 \\
\hline 0.90 & 4 & 3.9865 & 3.9303 & 3.8153 & 3.7515 & 3.6839 & 3.5015 & 3.5805 & 3.6368 & 3.0610 & 3.6731 \\
\hline 0.90 & 5 & 3.4961 & 3.4497 & 3.3544 & 3.2966 & 3.2348 & 3.2105 & 2.9167 & 3.0187 & 3.2117 & 3.1488 \\
\hline 0.90 & 6 & 3.1546 & 3.0983 & 3.0488 & 2.9954 & 2.9380 & 2.8428 & 2.7494 & 2.6193 & 2.8177 & 2.8043 \\
\hline 0.90 & 7 & 2.9122 & 2.8770 & 2.7767 & 2.6995 & 2.7270 & 2.5857 & 2.6252 & 2.6377 & 2.5401 & 2.5584 \\
\hline 0.90 & 8 & 2.7309 & 2.6877 & 2.6215 & 2.5512 & 2.4762 & 2.5130 & 2.3481 & 2.3904 & 2.3338 & 2.3739 \\
\hline 0.90 & 9 & 2.5801 & 2.5413 & 2.5003 & 2.4353 & 2.3657 & 2.3516 & 2.2950 & 2.2008 & 2.1740 & 2.2298 \\
\hline 0.90 & 10 & 2.4598 & 2.4243 & 2.3678 & 2.3422 & 2.2768 & 2.2240 & 2.2503 & 2.2469 & 2.3107 & 2.1137 \\
\hline 0.95 & 0 & 57.7180 & 56.6969 & 54.6527 & 50.5105 & 50.5640 & 52.7154 & 47.6037 & 42.4911 & 53.1138 & 63.7366 \\
\hline 0.95 & 1 & 13.1233 & 12.9222 & 12.5182 & 12.4144 & 11.7015 & 11.5919 & 10.5419 & 10.9966 & 9.8900 & 11.8680 \\
\hline 0.95 & 2 & 7.5278 & 7.4222 & 7.2090 & 6.8610 & 6.5094 & 6.8151 & 6.2408 & 5.6320 & 5.3215 & 6.3857 \\
\hline 0.95 & 3 & 5.5593 & 5.4474 & 5.2215 & 5.2290 & 5.0756 & 4.7596 & 4.7466 & 4.7113 & 4.8596 & 4.5615 \\
\hline 0.95 & 4 & 4.5600 & 4.4766 & 4.3623 & 4.2449 & 4.1238 & 4.0540 & 4.0012 & 3.6368 & 3.8171 & 3.6731 \\
\hline 0.95 & 5 & 3.9491 & 3.9029 & 3.7669 & 3.6688 & 3.5670 & 3.4192 & 3.2373 & 3.4572 & 3.2117 & 3.1488 \\
\hline 0.95 & 6 & 3.5477 & 3.4916 & 3.3770 & 3.2917 & 3.2025 & 3.1754 & 3.0050 & 2.9739 & 2.8177 & 2.8043 \\
\hline 0.95 & 7 & 3.2501 & 3.2015 & 3.1018 & 3.0256 & 2.9455 & 2.8609 & 2.8366 & 2.6377 & 2.9250 & 3.0482 \\
\hline 0.95 & 8 & 3.0289 & 2.9859 & 2.8972 & 2.8279 & 2.7544 & 2.7464 & 2.7084 & 2.6410 & 2.6670 & 2.8005 \\
\hline 0.95 & 9 & 2.8479 & 2.7993 & 2.7389 & 2.6749 & 2.6067 & 2.5540 & 2.4517 & 2.4208 & 2.4685 & 2.6088 \\
\hline 0.95 & 10 & 2.7041 & 2.6687 & 2.6127 & 2.5529 & 2.4889 & 2.4024 & 2.3882 & 2.2469 & 2.3107 & 2.4557 \\
\hline 0.99 & 0 & 88.1383 & 86.0673 & 84.0232 & 81.9790 & 75.7389 & 73.6945 & 63.3385 & 63.4716 & 53.1138 & 63.7366 \\
\hline 0.99 & 1 & 18.2712 & 17.7675 & 17.3645 & 16.9596 & 16.5526 & 16.1433 & 15.1091 & 14.0558 & 13.7457 & 11.8680 \\
\hline 0.99 & 2 & 10.0941 & 9.9229 & 9.5790 & 9.2329 & 8.6206 & 8.7969 & 8.2360 & 8.3211 & 7.0400 & 8.4480 \\
\hline 0.99 & 3 & 7.2133 & 7.1017 & 6.7979 & 6.6493 & 6.3403 & 6.3445 & 5.9462 & 5.5234 & 5.8892 & 5.8316 \\
\hline 0.99 & 4 & 5.7617 & 5.6786 & 5.4559 & 5.2305 & 5.2214 & 4.8799 & 4.8365 & 4.7740 & 4.5460 & 4.5805 \\
\hline 0.99 & 5 & 4.9170 & 4.8504 & 4.6741 & 4.5363 & 4.3954 & 4.2512 & 4.1877 & 3.8890 & 3.7734 & 3.8540 \\
\hline 0.99 & 6 & 4.3502 & 4.2779 & 4.16472 & 3.9820 & 3.8621 & 3.8379 & 3.5116 & 3.6659 & 3.2741 & 3.3812 \\
\hline 0.99 & 7 & 3.9395 & 3.8776 & 3.7517 & 3.6770 & 3.5993 & 3.4087 & 3.2557 & 3.2162 & 3.2971 & 3.0482 \\
\hline 0.99 & 8 & 3.6363 & 3.5820 & 3.4483 & 3.3804 & 3.3092 & 3.2113 & 3.0646 & 2.8874 & 2.9880 & 3.2004 \\
\hline 0.99 & 9 & 3.4034 & 3.3549 & 3.2557 & 3.1533 & 3.0872 & 3.0576 & 2.9161 & 2.8494 & 2.7510 & 2.9622 \\
\hline 0.99 & 10 & 3.2100 & 3.1574 & 3.0673 & 2.9738 & 2.9118 & 2.8460 & 2.7972 & 2.6279 & 2.5624 & 2.7713 \\
\hline
\end{tabular}

TABLE 5: OC values of sampling plans for $n=3, c=1$, and $\delta_{0}=2.0$.

\begin{tabular}{lccccccccc}
\hline$t_{0.5} / t_{0.5}^{0}$ & 1.0 & 1.5 & 2.0 & 2.5 & 3.0 & 3.5 & 4.0 & 4.5 & 5.0 \\
\hline OC & 0.0832 & 0.4276 & 0.6317 & 0.7464 & 0.8157 & 0.8602 & 0.8905 & 0.9120 & 0.9272 \\
\hline
\end{tabular}


is minimum, we can use the tables in this paper to pick up acceptance number $c$ and the upper bound in order to accept/reject the lot for a given probability of acceptance, say $p^{*}$. This paper also provides the sensitivity of the sampling plans in terms of the OC. We advise the industrial practitioner and the experimenter to adopt this plan in order to save the cost and time of the experiment. This plan can be further studied for many other distributions and various quality and reliability characteristics as future research.

\section{Acknowledgments}

The authors thank the editor and the reviewers for their helpful suggestions, comments, and encouragement, which helped in improving the final version of the paper.

\section{References}

[1] B. Epstein, "Truncated life tests in the exponential case," Annals of Mathematical Statistics, vol. 25, pp. 555-564, 1954.

[2] M. Sobel and J. A. Tischendrof, "Acceptance sampling with skew life test objective," in Proceedings of the 5th National Syposium on Reliability and Qualilty Control, pp. 108-118, Philadelphia, Pa, USA, 1959.

[3] H. P. Goode and J. H. K. Kao, "Sampling plans based on the Weibull distribution," in Proceedings of the 7th National Syposium on Reliability and Qualilty Control, pp. 24-40, Philadelphia, Pa, USA, 1961.

[4] S. S. Gupta and P. A. Groll, "Gamma distribution in acceptance sampling based on life tests," Journal of the American Statistical Association, vol. 56, pp. 942-970, 1961.

[5] S. S. Gupta, "Life test sampling plans for normal and lognormal distribution," Technometrics, vol. 4, pp. 151-175, 1962.

[6] F. W. Fertig and N. R. Mann, "Life-test sampling plans for twoparameter Weibull populations.," Technometrics, vol. 22, pp. 165-177, 1980.

[7] R. R. L. Kantam and K. Rosaiah, "Half Logistic distribution in acceptance sampling based on life tests," IAPQR Transactions, vol. 23, no. 2, pp. 117-125, 1998.

[8] A. Baklizi, "Acceptance sampling based on truncated life tests in the pareto distribution of the second kind," Advances and Applications in Statistics, vol. 3, pp. 33-48, 2003.

[9] C.-J. Wu and T.-R. Tsai, "Acceptance sampling plans for birnbaum-saunders distribution under truncated life tests," International Journal of Reliability, Quality and Safety Engineering, vol. 12, no. 6, pp. 507-519, 2005.

[10] K. Rosaiah and R. R. L. Kantam, "Acceptance sampling plans based on inverse Rayleigh distribution," Economic Quality Control, vol. 20, no. 2, pp. 277-286, 2005.

[11] K. Rosaiah, R. R. L. Kantam, and Ch. Santosh Kumar, "Reliability test plans for exponnetiated log-logistic distribution," Economic Quality Control, vol. 21, pp. 165-175, 2006.

[12] T.-R. Tsai and S.-J. Wu, "Acceptance sampling based on truncated life tests for generalized Rayleigh distribution," Journal of Applied Statistics, vol. 33, no. 6, pp. 595-600, 2006.

[13] N. Balakrishnan, V. Leiva, and J. López, "Acceptance sampling plans from truncated life tests based on the generalized Birnbaum-Saunders distribution," Communications in Statistics: Simulation and Computation, vol. 36, no. 3, pp. 643-656, 2007.
[14] G. Srinivasa Rao, M. E. Ghitany, and R. R. L. Kantam, "Reliability test plans for Marshall-Olkin extended exponential distribution," Applied Mathematical Sciences, vol. 3, no. 53-56, pp. 2745-2755, 2009.

[15] G. Srinivasa Rao, M. E. Ghitany, and R. R. L. Kantam, "An economic reliability test plan for Marshall-Olkin extended exponential distribution," Applied Mathematical Sciences, vol. 5, no. 1-4, pp. 103-112, 2011.

[16] M. Aslam, C.-H. Jun, and M. Ahmad, "A group sampling plan based on truncated life test for gamma distributed items," Pakistan Journal of Statistics, vol. 25, no. 3, pp. 333-340, 2009.

[17] G. Srinivasa Rao, M. E. Ghitany, and R. R. L. Kantam, "Reliability test plans for Marshall-Olkin extended exponential distribution," Applied Mathematical Sciences, vol. 3, no. 53-56, pp. 2745-2755, 2009.

[18] Y. L. Lio, T.-R. Tsai, and S.-J. Wu, "Acceptance sampling plans from truncated life tests based on the birnbaum-saunders distribution for percentiles," Communications in Statistics: Simulation and Computation, vol. 39, no. 1, pp. 119-136, 2010.

[19] G. Srinivasa Rao and R. R. L. Kantam, "Acceptance sampling plans from truncated life tests based on log-logistic distribution for percentiles," Economic Quality Control, vol. 25, no. 2, pp. 153$167,2010$.

[20] A. Wood, "Predicting software reliability," IEEE Transactions on Software Engineering, vol. 22, pp. 69-77, 1996.

[21] M. V. Aarset, "How to identify a bathtub hazard rate," IEEE Transactions on Reliability, vol. 36, no. 1, pp. 106-108, 1987. 

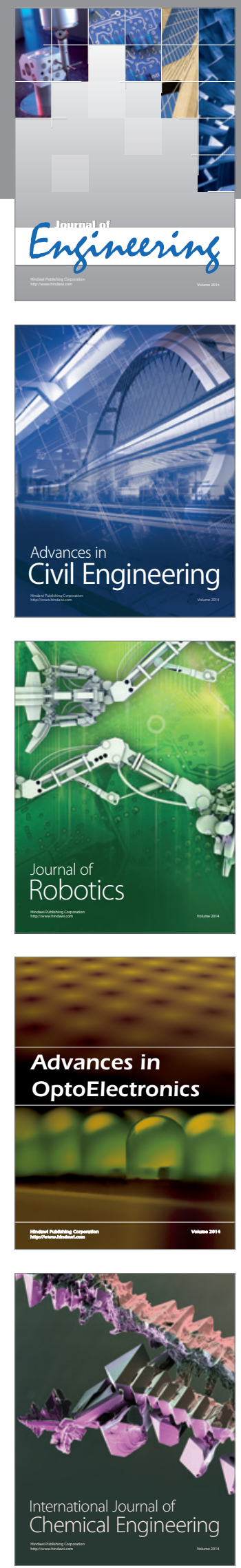

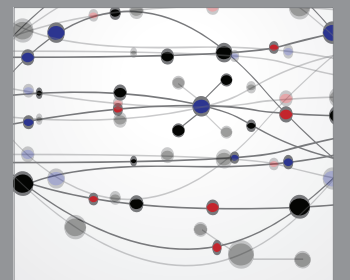

The Scientific World Journal
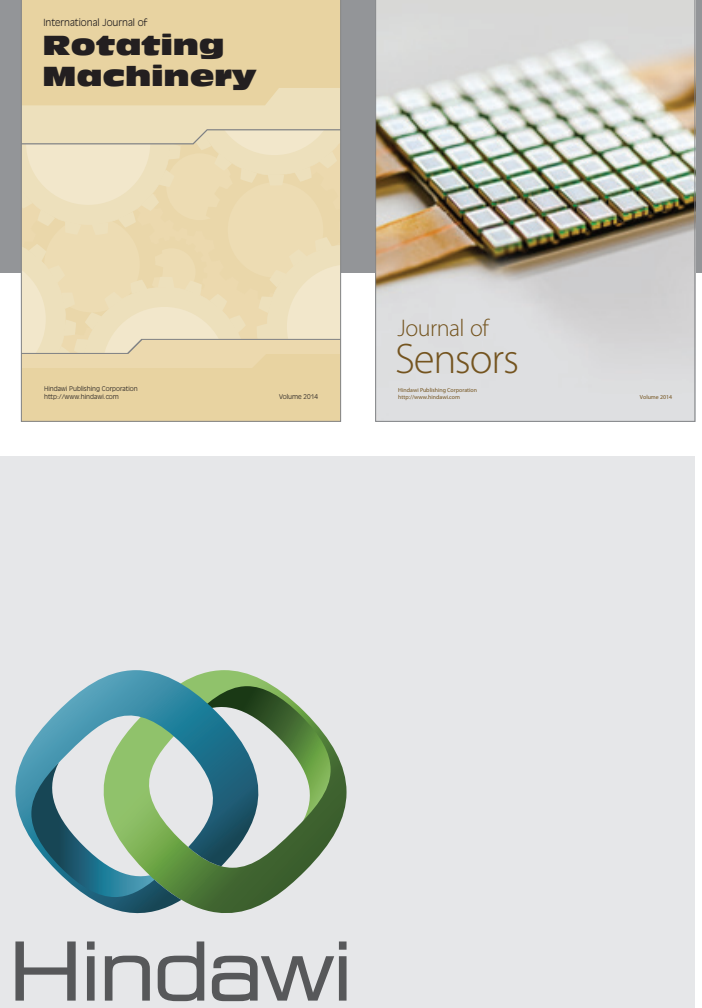

Submit your manuscripts at http://www.hindawi.com
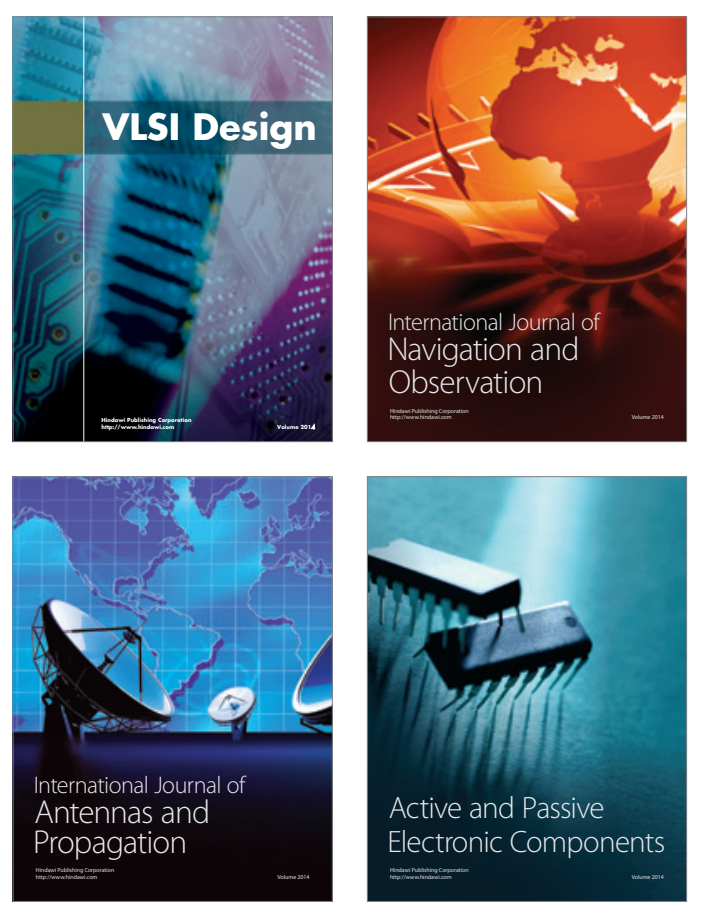
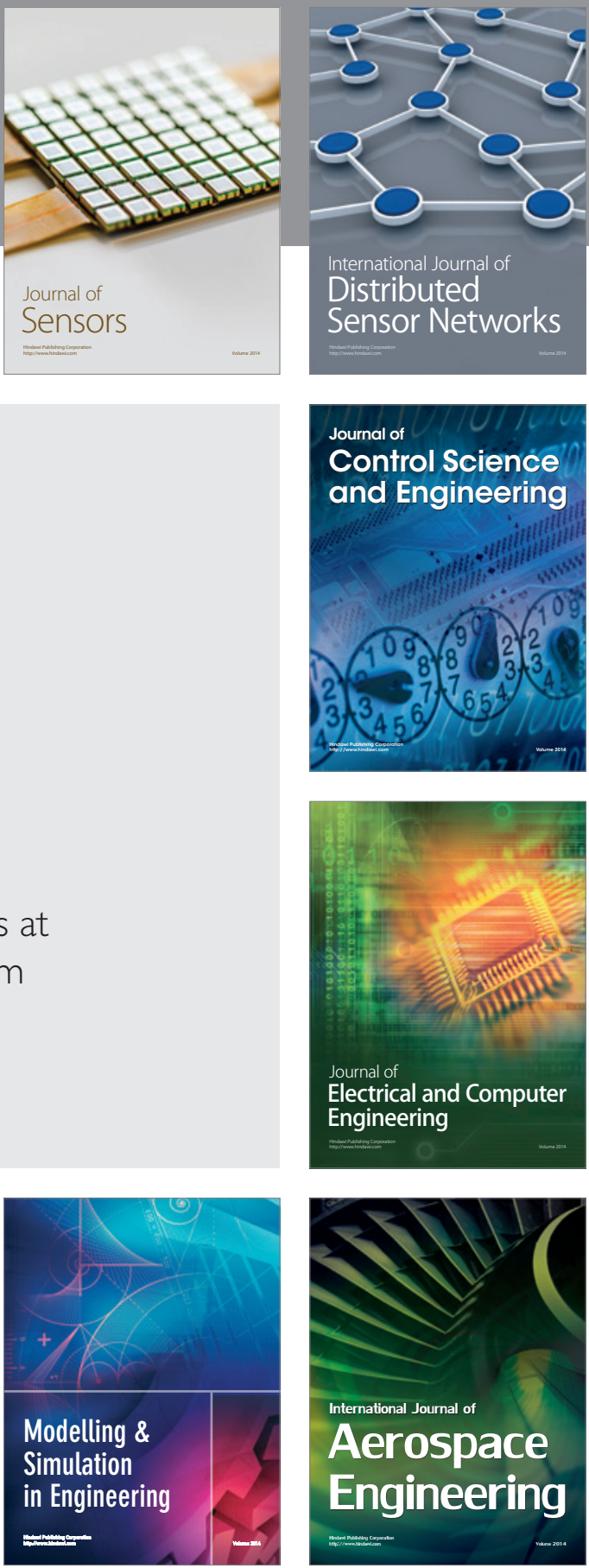

Journal of

Control Science

and Engineering
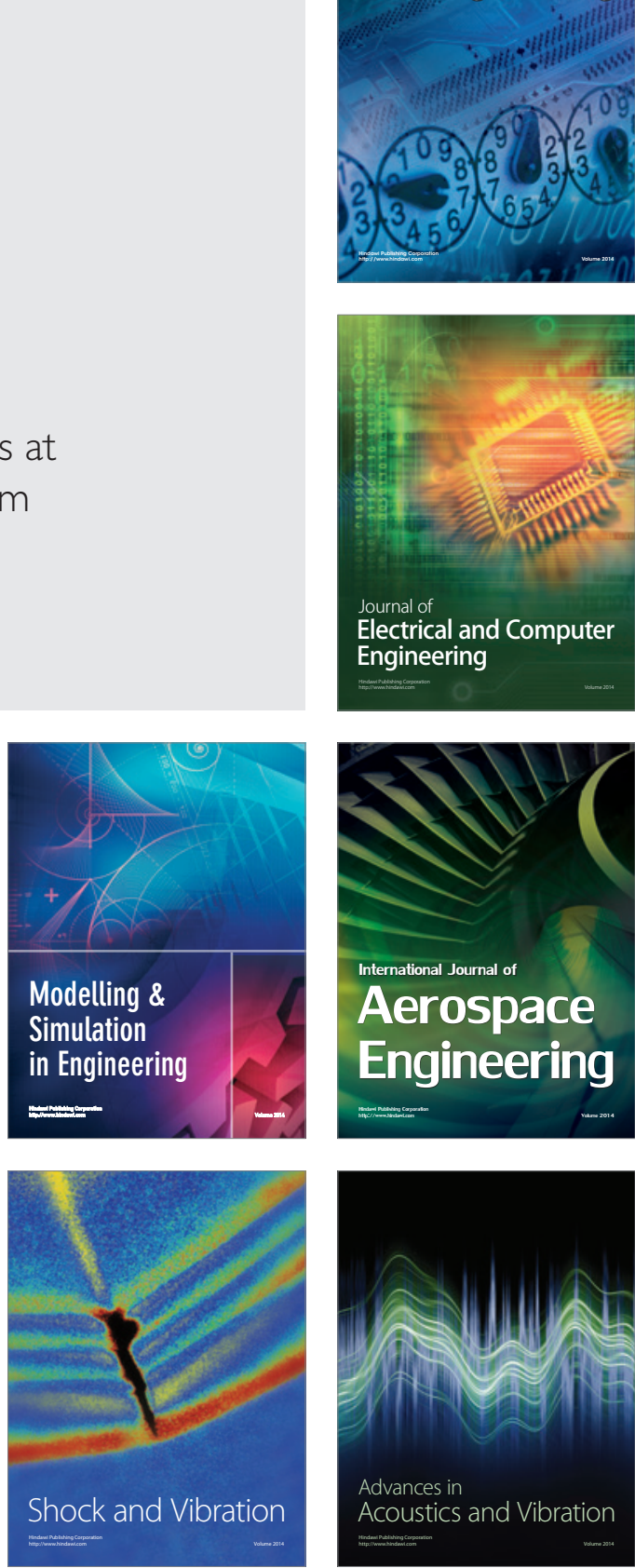